\title{
Obesity in Gestational Diabetes: Emerging Twin Challenge for Perinatal Care in India
}

\author{
${ }^{1}$ Tarakeswari Surapaneni, ${ }^{2}$ Evita Fernandez \\ ${ }^{1}$ Consultant, Obstetric Medicine and High-risk Obstetrics, Fernandez Hospital Pvt Ltd, Bogulkunta, Hyderabad, Andhra Pradesh, India \\ ${ }^{2}$ Consultant Obstetrician, Fernandez Hospital Pvt Ltd, Bogulkunta, Hyderabad, Andhra Pradesh, India
}

Correspondence: Tarakeswari Surapaneni, Consultant, Obstetric Medicine and High-risk Obstetrics, Fernandez Hospital Pvt Ltd, Bogulkunta, Hyderabad-500001, Andhra Pradesh India, Phone: 91-40-40222309/447/427, Fax: 91-40-24753482 e-mail: tarakeswari@gmail.com

\section{ABSTRACT}

The current study aimed to determine the prevalence of obesity and gestational diabetes (GDM) and the association of obesity and gestational diabetes with adverse pregnancy outcomes in a population of pregnant women. Routine antenatal care included the estimation of body mass indices, assessment of blood glucose levels, including glucose challenge tests and oral glucose tolerance test, fetal growth monitoring and nutritional counseling. The prevalence of GDM and obesity in this population was $8.43 \%$ (95\% Cl: $7.47-9.40)$ and $19.49 \%$ (95\% Cl: $18.12-20.87)$ respectively. The prevalence of obesity increased to $54.63 \%$ (95\% Cl: $52.91-56.36)$, if we used the ICMR guidelines for BMI in this population. Cesarean sections (adjusted OR: 2.04, 95\% Cl: 1.43-2.89), large for gestational age (LGA) babies (adjusted OR: 3.82, 95\% Cl: 2.11-6.92) and macrosomia (adjusted OR: 20.90, 95\% Cl: 3.29-132.77) was associated with obesity in GDM (based on the ICMR guidelines for BMI). Results from this study indicate that gestational diabetes and obesity are increasingly important priorities for perinatal care in India.

Keywords: Gestational diabetes, Increased cesarean section rate, Obesity, Pregnancy, India.

\section{INTRODUCTION}

India is in a state of epidemiological transition with an increasing prevalence of noncommunicable diseases. An estimated 79 million people are expected to have diabetes mellitus in India by the year $2030 .{ }^{1}$ The prevalence of gestational diabetes (GDM) in India varies from $9.9 \%$ in rural areas to $17.8 \%$ in an urban population. ${ }^{2}$ The National Family Health Survey (NFHS)III (2005-06) of India reports an increasing prevalence (14.8\%) of overweight or obesity among women aged 15 to 49 years (ranging from $28.9 \%$ in urban areas to $8.6 \%$ in rural areas) compared to $10.6 \%$ in $1998-99 .{ }^{3}$ Gestational diabetes and obesity have adverse consequences on outcomes of pregnancy, including cesarean delivery, perinatal deaths, pre-eclampsia, birth defects, macrosomia, and morbidity associated with subsequent childhood obesity. ${ }^{4-18}$ In this manuscript, we report on the prevalence of gestational diabetes and obesity and the outcomes of pregnancy in a pregnant population with obesity and GDM at an advanced tertiary care center in South India.

\section{METHODS}

We did a retrospective cohort study of pregnant women, who were booked for delivery at Fernandez Hospital, an advanced tertiary care perinatal institute in South India during the year 2007. The study protocol was approved by the instituitional

Date of Received: 06-09-10

Date of Acceptance: 26-09-10

Date of Publication: Sep. 2010 review board and adhered to the tenets of the declaration of Helsinki. Women with multifetal pregnancies, referrals from other hospitals, patients who came only for delivery at the institute and who were not assessed at Fernandez Hospital through the antenatal period, and late bookings after 34 weeks were excluded from this study. All women with incomplete data were also excluded from this study.

Each pregnant woman, who registers in the first trimester for an antenatal check up at Fernandez Hospital undergoes a standardized examination protocol. This includes collecting details of maternal age at registration, parity of the mother, details of previous miscarriages, if any and prior obstetric history, and history of any medical complication especially hypertension, diabetes and hypothyroidism. The presence of medical problems prior to registration is verified from the medical records of the patient and confirmed based on a clinical diagnosis after examination by a physician. A complete physical workup is repeated at registration, including assessment of blood pressure, height and weight. A standard measuring tape was used to measure the height of subjects up to one decimal place. A standard electronic platform weighing scale was used to measure weight $(\mathrm{kg})$ to the nearest one decimal place correcting for the zero-error. Body mass indices (BMI) were derived and individuals were categorized as underweight $\left(<18.5 \mathrm{~kg} / \mathrm{m}^{2}\right)$, normal $\left(18.5-24.9 \mathrm{~kg} / \mathrm{m}^{2}\right)$, overweight $\left(25.0-29.9 \mathrm{~kg} / \mathrm{m}^{2}\right)$ and obese $\left(\geq 30 \mathrm{~kg} / \mathrm{m}^{2}\right)$. Each woman identified as obese was offered nutritional counseling and a customized nutritional plan as part of the antenatal care. 
All women at risk for GDM (with family history of diabetes, previous history of GDM) were subjected to early screening at booking. Universal screening for gestational diabetes included a $50 \mathrm{gm}$ glucose challenge test (in the second trimester of pregnancy) with a 2 hours post glucose cutoff of $140 \mathrm{mg} / \mathrm{dl}$ and confirmed by a $75 \mathrm{gm}$ oral glucose tolerance test using the modified WHO criteria (75 gm glucose fasting $95 \mathrm{mg} / \mathrm{dl}$ and 2 hours $155 \mathrm{mg} / \mathrm{dl}) .{ }^{19}$ All pregnant women were offered first trimester ultrasound screening to assess gestational age, nuchal fold thickness and presence of structural abnormalities at 11 to $13^{+6}$ weeks, a targetted imaging for fetal anomalies at 18 to 22 weeks and a fetal growth assessment at 32 to 34 weeks.

We defined gestational diabetes (GDM) as carbohydrate intolerance with onset or first recognition during pregnancy. We defined pregnancy induced hypertension to include preeclampsia or eclampsia. We defined pre-eclampsia as new onset hypertension after 20 weeks gestation with proteinuria of $1^{+}$or more. Severe pre-eclampsia was diagnosed, if proteinuria was $2^{+}$or more, severe HTN (sBP $\geq 160 \mathrm{mmHg}$ and $\mathrm{dBP} \geq$ $110 \mathrm{~mm}$ of $\mathrm{Hg}$ ) or if there was any evidence of organ involvement, imminent symptoms, thrombocytopenia, HELLP syndrome or IUGR. Eclampsia was diagnosed, if convulsions developed in a woman with pre-eclampsia. Intrapartum complications of interest included postpartum hemorrhage $(\mathrm{PPH})$ and obstetric anal sphincter injury (OASI). We defined postpartum hemorrhage as excessive blood loss post delivery (more than $500 \mathrm{ml}$ after a vaginal delivery and more than $1000 \mathrm{ml}$ after a cesarean section) within the first 24 hours of delivery and requiring additional uterotonics, procedures or interventions. Obstetric anal sphinter injuries was a term used for Grade III A/B and Grade IV perineal injury post-vaginal delivery. Preterm delivery was defined as delivery before 37 completed weeks. The babies were classified at birth as appropriate for gestational age (AGA), small for gestational age (SGA) and large for gestational age (LGA) at birth using Lubchenco charts. Intrauterine growth restriction was defined based on population growth curves for Indian women (Mediscan Systems, Chennai).

\section{STATISTICAL ANALYSIS}

We excluded severe outliers of BMI from the analysis, the severe outliers are determined by the following formula 'Severe outlier $=\mathrm{X}<\mathrm{Q}(25)-3 \mathrm{IQR}$ or $\mathrm{X}>\mathrm{Q}(75)+3 \mathrm{IQR}$ ' where Q25 is the 25th percentile, Q75 is the 75th percentile, IQR (Interquartile Range $)=75$ th percentile-25th percentile and $\mathrm{X}$ is value of BMI. We compared the characteristics of women in the three groups of interest (normal, GDM with obesity, GDM without obesity) using analysis of variance test (ANOVA) for continuous variables and a chi-square or Fisher's exact test for categorical variables. We used a bivariate analysis followed by a multivariate logistic regression model to explore for associations of the three groups with different outcomes of interest. Potential associations are expressed using odds ratios (OR) and 95\% confidence intervals (95\% CI) around the point estimates. We considered a p-value of $<0.05$ as statistically significant for this analysis. We used STATA Version 9.0 (College Station, Texas, USA) for the statistical analysis. We also recategorized subjects based on the recent Indian Council for Medical Research (ICMR) guidelines for obesity in an Indian population (BMI < 18.4 is underweight, BMI 18.5-22.9 is normal, BMI 23-24.9 is overweight and BMI $>25$ is obese).

\section{RESULTS}

Four thousand five hundred and twenty-four deliveries were conducted at Fernandez Hospital between January 2007 and December 2007. We excluded 1013 cases because of late registrations, referrals for delivery from other hospitals and walk in deliveries as information on antenatal checks was not available. An additional 228 women were excluded because information on BMI was incomplete or missing and an additional 13 women were excluded because the BMI values were considered as severe outliers. Sixty-nine women, whose BMI was below normal were also excluded from the analysis. Table 1 shows the characteristics of the 3, 201 pregnant women included in the study.

The prevalence of GDM and obesity in this population was 8.43\% (95\% CI: 7.47-9.40) and 19.49\% (95\% CI: 18.12-20.87) respectively. The prevalence of obesity increased to $54.63 \%$ (95\% CI: 52.91-56.36), if we used the ICMR guidelines in this population. One hundred and six women (3.31\%, 95\% CI: 2.693.93) had both obesity and GDM. An additional 99 women (3.09\%, 95\% CI: 2.49-3.69) had GDM and was overweight. If we use the ICMR guidelines for obesity, 203 (6.34\%, 95\% CI: 5.50-7.19) women had GDM and obesity and an additional 29 (0.91\%, 0.58-1.23) women had GDM and were overweight.

Women with GDM were older compared to women without GDM (mean age 28.52 (5.03) years and 25.85 (4.07) years respectively, ANOVA $\mathrm{F}=102.06, \mathrm{p}<0.001)$. After adjusting for the age of the mother, gestational diabetes was associated with overweight (adjusted OR 1.74, 95\% CI: 1.24-2.44) and obesity (adjusted OR 3.81, 95\% CI: 2.66-5.45) but was not associated with gestational hypertensive disorders (adjusted OR 1.14, 95\% CI: 0.73-1.77). If we use the ICMR guidelines for

Table 1: Characteristics of the 3, 201 pregnant women in the study

$\begin{array}{lr}\text { Mean age (SD) in years } & 26.07(4.23) \\ \text { Median age, range (years) } & 26.00,18 \text { to } 46 \\ \text { Nulliparous } & 1,681(52.51 \%) \\ \text { Miscarriages } & 829(25.90 \%) \\ \text { Normal BMI } & 1,452(45.36 \%) \\ \text { Overweight BMI } & 1,125(35.15 \%) \\ \text { Obese BMI } & 624(19.49 \%) \\ \text { ICMR-normal BMI } & 915(28.58 \%) \\ \text { ICMR-overweight BMI } & 537(16.78 \%) \\ \text { ICMR-obese BMI } & 1,749(54.64 \%) \\ \text { Chronic hypertension } & 43(1.34 \%) \\ \text { Hypothyroid } & 183(5.72 \%) \\ \text { Diabetes mellitus } & 51(1.59 \%) \\ \text { Gestational hypertension } & 273(8.53 \%) \\ \text { Gestational diabetes } & 270(8.43 \%)\end{array}$


BMI, GDM was associated with obesity (age adjusted OR 2.61, 95\% CI: 1.77 to 3.85) but was not associated with overweight (age adjusted OR 1.30, 95\% CI: 0.77 to 2.19).

We compared the intrapartum events and outcomes of pregnancy (Table 2) between three groups-a normal group with normal BMI and no associated medical conditions ( $\mathrm{n}=1,316$ ), a group with gestational diabetes and obesity $(\mathrm{n}=106)$ and a group of gestational diabetes without obesity $(\mathrm{n}=164)$ and repeated the analysis using the recent ICMR guidelines for BMI in India (Table 3).

In a regression analysis that adjusted for maternal age and based on conventional definitions of BMI, cesarean sections were more likely in the GDM without obesity (adjusted OR: 1.57, 95\% CI: 1.11-2.22) and GDM with obesity (adjusted OR: 2.04, 95\% CI: 1.32-3.17) compared to the group of normal women. If we use the ICMR guidelines for BMI and normal women as the reference group, cesarean section was associated with GDM and obese women (adjusted OR: 2.04\%, 95\% CI: 1.43-2.89) but was not associated with GDM and overweight (adjusted OR: 1.04, 95\% CI: 0.61-1.78).

Compared to normals and based on conventional BMI categories, preterm deliveries were more likely in the GDM with obesity group (maternal age adjusted OR 1.92, 95\% CI: 1.08-3.39) but was not significantly different in the GDM without obesity group (maternal age adjusted OR: 1.37, 95\% CI: 0.85-2.21). If we use the ICMR guidelines for BMI and normal women as the reference, preterm deliveries were not associated with either GDM and obesity (maternal age adjusted OR: 1.56 , 95\% CI: $0.97-2.51$ ) or just GDM (maternal age adjusted OR: 1.24, 95\% CI: 0.58-2.62).

Compared to normals and based on conventional BMI categories, large for gestational age (LGA) babies were more likely in the GDM with obesity group (maternal age adjusted OR: 5.90, 95\% CI: 3.04-11.42) and GDM without obesity group (maternal age adjusted OR: 2.36, 95\% CI: 1.27 to 4.39). If we use the ICMR guidelines for BMI and normal women as the reference, LGA was associated only with GDM and obesity (maternal age adjusted OR: 3.82, 95\% CI: 2.11-6.92) and not with GDM and no obesity (maternal age adjusted OR: 2.24, 95\% CI: 0.78-6.42).

Compared to normals and based on conventional BMI categories, macrosomia (birthweight $>4 \mathrm{~kg}$ ) was significantly higher in the GDM with obesity group (maternal age adjusted OR: 32.71, 95\% CI: 7.95-134.63) but was not significantly higher in the GDM without obesity group (maternal age adjusted OR: 1.06, 95\% CI: 0.11-10.22). If we use the ICMR guidelines for BMI and normal women as the reference, macrosomia was associated only with GDM and obesity (maternal age adjusted OR: 20.90, 95\% CI: 3.29-132.77) and not with GDM and no obesity (maternal age adjusted OR: 4.97, 95\% CI: 0.24-101.07).

\section{DISCUSSION}

Results from this study indicate that gestational diabetes and obesity are increasingly important priorities for perinatal care

Table 2: Intrapartum events and outcomes of pregnancy in the study population

\begin{tabular}{|c|c|c|c|c|}
\hline Characteristics & $\begin{array}{c}\text { Normal } \\
(n=1,316)\end{array}$ & $\begin{array}{c}\text { GDM without } \\
\text { obesity } \\
(n=164)\end{array}$ & $\begin{array}{l}\text { GDM with } \\
\text { obesity } \\
(n=106)\end{array}$ & $\begin{array}{c}\text { Statistical } \\
\text { significance }\end{array}$ \\
\hline Mean (SD) gestational age & $38.26(1.98)$ & $37.43(2.04)$ & $37.45(1.91)$ & ANOVA $F=19.27, P<0.001$ \\
\hline Cesarean section & $482(36.63 \%)$ & $89(54.27 \%)$ & $64(60.38 \%)$ & Chi-square test $\mathrm{P}<0.001$ \\
\hline Obstetric anal sphincter injury & $11(0.84 \%)$ & $0(0.0 \%)$ & $0(0.0 \%)$ & Fishers exact test $P=0.68$ \\
\hline Postpartum hemorrhage & $53(4.03 \%)$ & $2(1.22 \%)$ & $3(2.83 \%)$ & Fishers exact test $P=0.18$ \\
\hline Mean (SD) birthweight in kg & $2.90(0.50)$ & $2.88(0.58)$ & $3.14(0.63)$ & ANOVA $F=11.61, p<0.001$ \\
\hline Preterm delivery & $146(11.09 \%)$ & $27(16.46 \%)$ & $21(19.81 \%)$ & Fishers exact test $p=0.008$ \\
\hline Large for gestational age babies & $43(3.27 \%)$ & $16(9.76 \%)$ & $24(22.64 \%)$ & Chi-square test $p<0.001$ \\
\hline Macrosomia (Birthweight > 4 kg) & $6(0.46 \%)$ & $1(0.61 \%)$ & $11(10.38 \%)$ & Fishers exact test $p<0.001$ \\
\hline Still born & $17(1.29 \%)$ & $1(0.61 \%)$ & $0(0.0 \%)$ & Fishers exact test $p=0.70$ \\
\hline Neonatal intensive & $83(6.31 \%)$ & $11(6.71 \%)$ & $14(13.21 \%)$ & Chi-square test $p=0.03$ \\
\hline Care unit admission & & & & \\
\hline
\end{tabular}

Table 3: Intrapartum events and outcomes of pregnancy in the study population based on the ICMR guidelines for body mass index in India

\begin{tabular}{|c|c|c|c|c|}
\hline Characteristics & $\begin{array}{l}\text { Normal } \\
(n=878)\end{array}$ & $\begin{array}{c}\text { GDM without } \\
\text { obesity } \\
(n=65)\end{array}$ & $\begin{array}{c}\text { GDM with } \\
\text { obesity } \\
(n=203)\end{array}$ & $\begin{array}{c}\text { Statistical } \\
\text { significance }\end{array}$ \\
\hline Cesarean section & $311(35.42 \%)$ & $26(40.00 \%)$ & $125(61.58 \%)$ & Chi-square test $P<0.001$ \\
\hline Obstetric anal sphincter injury & $6(0.68 \%)$ & $0(0.0 \%)$ & $0(0.0 \%)$ & Fishers exact test $P=0.72$ \\
\hline Postpartum hemorrhage & $33(3.76 \%)$ & $1(1.54 \%)$ & $4(1.95 \%)$ & Fishers exact test $\mathrm{P}=0.47$ \\
\hline Preterm delivery & $99(11.28 \%)$ & $9(13.85 \%)$ & $39(19.02 \%)$ & Fishers exact test $p=0.01$ \\
\hline Large for gestational age babies & $31(3.53 \%)$ & $5(7.69 \%)$ & $34(17.08 \%)$ & Fishers exact test $p<0.001$ \\
\hline Macrosomia (Birthweight > 4 kg) & $3(0.34 \%)$ & $1(1.54 \%)$ & $11(5.37 \%)$ & Fishers exact test $p<0.001$ \\
\hline Still born & $10(1.14 \%)$ & $1(1.54 \%)$ & $0(0.0 \%)$ & Fishers exact test $p=0.22$ \\
\hline Neonatal intensive care unit admission & $66(7.52 \%)$ & $3(4.62 \%)$ & $22(10.73 \%)$ & Chi-square test $p=0.22$ \\
\hline
\end{tabular}


in India. Results from the study suggest that the incidence of obese and overweight pregnant women in urban India may be similar to that quoted in the Confidential Enquiry into Maternal and Child Health (CEMACH), 2007. ${ }^{20}$ One in five pregnant women was obese and nearly one in ten pregnant women had gestational diabetes. An estimated 3.31\% of women had both gestational diabetes and obesity. However, these estimates rise dramatically, if we use the recent ICMR guidelines for BMI. Based on the ICMR guidelines, over half of pregnant women attending this tertiary care center were obese and $6.34 \%$ of women had GDM and obesity. Women with gestational diabetes and obesity were more likely to have cesarean sections, large of gestational age babies and babies with a birthweight $>4 \mathrm{~kg}$.

These results and the revised cutoffs for BMI proposed by the ICMR have several important implications for the perinatal care structure in India. Viewed within the context of an increasing population of women with obesity in the reproductive age group and diabetes mellitus in the general population, the perinatal care structure in India will have to focus on an increased need or demand for cesarean sections and consequent perioperative and postoperative management, an increased need for neonatal care facilities, and better postpartum management structures. The need for improved postpartum management structures is especially important considering that approximately $40 \%$ of women with GDM will go on to develop type II diabetes. ${ }^{21,22}$ Additionally, the NFHS-III reports that only 36.8\% of pregnant women received postpartum care from any health care personnel within 2 days of delivery. ${ }^{3}$ The consequence of maternal diabetes and obesity on the long-term health of the child is another important consideration.

It is possible that some proportion of the women diagnosed with GDM may actually be previously undiagnosed diabetes mellitus especially as pregnancy may be the first contact with the health system. Although, all women diagnosed with GDM are advised to have an OGTT in 6 weeks postpartum, we are unable to report on the postpartum blood sugar levels after OGTT in 6 weeks or 12 weeks as the majority of patients are referrals (considering the study center is an advanced tertiary care center) and prefer the postpartum follow ups with their primary physicians. Although telephonic interviews were attempted, this was not followed up as we could not ascertain conclusively the test method used for OGTT, if done, or if the lab followed a standard protocol. It is possible that a proportion of women diagnosed with GDM may not have a return to normal blood sugar levels in 6 weeks either because the treatment regime is not adhered to or because they had a chronic DM that was misclassified as GDM. Perinatal care structures in India will also have to focus on developing and improving internatal and preconception care including the development of standardized management protocols or appropriate adaptation of existing protocols to address issues pertaining to care in the postpartum and internatal care. The concept of preconception care and internatal care is currently not well recognized in India with almost non-existent health systems that focus on these two areas.
The generalizability of these results is a limitation of the study. The study population accessed an advanced tertiary care referral perinatal center and may not be representative of the general pregnant population of India. However, given the increasing prevalence of obesity and diabetes in India, these results support the need to consider the spectrum of diabetes and obesity as important priorities for perinatal care in India. This will need a major paradigm shift from the current infectious and mortality focused perinatal care to include a morbidity focused clinical care that will be longer term and more cost intensive. This will also need to translate into the development of standardized cost efficient protocols that can be implemented at the primary and secondary care levels in India.

\section{CONCLUSION}

Gestational diabetes and obesity are public health problems in India with consequences on the type of delivery and fetal growth. Postpartum follow-up for these conditions have to adhere to a stricter and standardized protocol especially as postpartum and internatal care do not receive the same attention as antenatal care. The lack of adequate postpartum care or internatal care protocols involving primary physicians will probably lead to complications in the subsequent pregnancy. The revised guidelines proposed by the ICMR for BMI in India will increase the prevalence of pregnant women with obesity in India with a consequent increase in the prevalence of pregnant women with gestational diabetes and obesity and a consequent increase in the number of neonates that need additional care.The increased prevalence will translate to altered management protocols for a significant number of pregnant women and place an additional strain on the perinatal health structure in India.

\section{REFERENCES}

1. Wild S, Roglic G, Green A, Sicree R, King H. Global prevalence of diabetes. Diabetes care 2004;27:1047-53.

2. Seshiah V, Balaji V, Balaji SM, Sanjeevi CB, Green A. Gestational Diabetes Mellitus in India. The journal of the association of physicians of India 2004;52:707-11.

3. National family health survey-III India. Accessed online at http:/ /www.nfhsindia.org/pdf/India.pdf on June 27, 2010.

4. HAPO study cooperative research group hyperglycemia and adverse pregnancy outcomes. N Engl J Med 2008;358:19912002.

5. Raatikainen, Kaisa, Heiskanen N, Heinonen S. Transition from overweight to obesity worsens pregnancy outcome in a bmidependent manner. Obesity 2006;14:165-71.

6. Cedergren MI, Maternal morbid obesity and the risk of adverse pregnancy outcome. Obstet gynecol 2004;103:219-24.

7. Honor Wolfe, High prepregnancy body-mass index: A maternal-fetal risk factor. N Engl J Med, Jan 15, 1998; 338(3)191-92.

8. Cnattingius S, Bergström R, Lipworth L, Kramer MS. Prepregnancy weight and the risk of adverse pregnancy outcomes. N Engl J Med 1998;338:147-52.

9. Watkins ML, Rasmussen SA, Honein MA, Botto LD, Moore CA. Maternal obesity and risk for birth defects pediatrics 2003;111;1152-58. 
10. Bujold E, Hammoud A, Schild C, Krapp M, Baumann P. The role of maternal body mass index in outcomes of vaginal births after cesarean. American journal of obstetrics and gynecology 2005;193:1517-21.

11. Langer O, Yogev Y, Elly MJ, Xenakis EMJ, Brustman L. Overweight and obese in gestational diabetes: The impact on pregnancy outcome. American journal of obstetrics and gynecology 2005;192:1768-76.

12. LaCoursiere DY, Bloebaum L, Duncan JD, Varner MW. Population-based trends and correlates of maternal overweight and obesity, Utah 1991 to 2001. American journal of obstetrics and gynecology 2005;192:832-39.

13. Weiss JL, Malone FD, Emig D, Ball RH, Nyberg DA, Comstock $\mathrm{CH}$, et al. Obesity, obstetric complications and cesarean delivery rate. A population-based screening study. American journal of obstetrics and gynecology 2004;190:1091e7.

14. Goodall PT, Ahn JT, Chapa JB, Hibbard JU. Obesity as a risk factor for failed trial of labor in patients with previous cesarean delivery. American journal of obstetrics and gynecology 2005;192:1423-26.

15. Sukalich S, Mingione MJ, Glantz JC. Obstetric outcomes in overweight and obese adolescents. American journal of obstetrics and gynecology 2006;195:851-55.
16. Ramos GA, Caughey AB. The inter-relationship between ethnicity and obesity on obstetric outcomes, American journal of obstetrics and gynecology 2005;193:1089-93.

17. Lombardi DG, Barton JR, O’Brien JM, Istwan NK, Sibai BM. Does an obese prepregnancy body mass index influence outcome in pregnancies complicated by mild gestational hypertension remote from term? American Journal of Obstetrics and Gynecology 2005;192:1472-74.

18. A Kumari. Pregnancy outcome in women with morbid obesity. International journal of gynecology and Obstetrics, 73(2):10107.

19. WHO and international diabetes federation 1999.

20. Confidential enquiry into maternal and child health. Perinatal mortality 2005: England, Wales and Northern Ireland. CEMACH: London 2007.

21. Conway DL, Langer O: Effects of new criteria for type 2 diabetes on the rate of postpartum glucose intolerance in women with gestational diabetes. Am J Obstet Gynecol 1999;181:610-14.

22. Schaefer-Graf UM, Buchanan TA, Xiang AH, Peters RK, Kjos SL: Clinical predictors for a high-risk for the development of diabetes mellitus in the early puerperium in women with recent gestational diabetes mellitus. Am J Obstet Gynecol, 2002;186: 751-56. 